North-Holland, Amsterdam

Section V. Other detectors

\title{
BUBBLE CHAMBER FOR FIXED-TARGET EXPERIMENTS AT MULTI-TeV ACCELERATORS
}

\author{
Gert G. HARIGEL
}

CERN, Geneva, Switzerland

A tentative layout for a huge argon bubble chamber/calorimeter/scintillation detector, to be used for neutrino experiments at a multi-TeV accelerator, is described. Such a detector can be operated in beams with various spill duration and it combines the features of a bubble chamber with properties of counter detectors.

\section{Introduction}

A large argon bubble chamber/calorimeter could become a useful detector for fixed-target physics at multi-TeV accelerators. The idea to use liquid argon was first outlined in 1978 for Fermilab Tevatron neutrino physics [1]. Subsequently various aspects have been studied experimentally in a small test device [2-8], and one potential application for non-accelerator physics was described in more detail [9]. Here, we limit ourselves to a discussion of tentative design features of a large chamber for neutrino physics, taking into account the boundary conditions imposed by a multi-TeV hadron collider, which could be operated for our purpose either with regular (dedicated and expensive) or alternate (parasitic) extraction techniques. A bubble chamber filled with liquid argon can be conveniently adapted to most of these unique conditions. Our approach to neutrino experiments with a bubble chamber at TeV-energies differs from ideas proposed earlier [10]. The physics interest in fixed-target experiments at very high energies has been outlined elsewhere in these proceedings [11].

\section{Characteristics of an argon bubble chamber for TeV-neutrino physics}

\subsection{The bubble chamber picture}

A bubble chamber photograph provides a good vertex recognition, in particular it allows the track multiplicity to be counted, to discriminate electrons at the vertex from downstream gammas, and to identify strange particles. The required optical resolution can be obtained by high-resolution photography (holography) and is improved by a magnetic field, which frees the forward cone of the vertex region from low energy particles.
Muon identification, without the usual help of additional counters, is possible inside the chamber liquid, provided the chamber is at least several interaction lengths long.

The centre of gravity of showers (electromagnetic or hadronic) can be determined graphically from the stereo-photographs. The visual measurement of the angular shower direction is superior to a determination from calorimetry alone, and might be somewhat better in the absence rather than in the presence of a magnetic field.

The bubble chamber photograph helps to interpret correctly the calorimeter measurements and to disentangle hadronic from electromagnetic showers.

The sign of the electric charge of muons - and probably of many primary electrons - can be determined from the photograph if sufficient magnetic field strength and chamber length are available.

Particle identification $(\pi, K, p)$ from bubble densities is limited essentially to momenta smaller than 1 $\mathrm{GeV} / c$, i.e. mainly to tracks from secondary or tertiary interactions. It has not yet been experimentally verified, if the relativistic rise of ionization causes sufficient change in bubble densities in liquid argon to identify particles at very high energies.

\subsection{Calorimetry from charge collection}

Calorimetry via charge collection in an electric field is required primarily for the energy measurement of heavy showers, rather than for single tracks. Momentum determination from track curvature in a magnetic field at very high momenta is rendered almost impossible due to the short track length before secondary interaction takes place and showers develop. Reliable measurements from charge collection call for careful monitoring of the purity of argon, to be done either continuously by an in-built $\beta$-source or by a hadron beam of known intensity and momentum. 


\subsection{Scintillatıon}

The main application of the scintillation pulse, produced by a bunch of particles, is as an event trigger for the bubble chamber cameras, saving film costs when event rates are low. The discrimination level for such a signal has to be well above the one from single through-going muons or cosmic rays.

The fast, intense scintillation pulse is very useful for various other purposes: it is a unique time trigger, which allows:

- the bubble chamber to be operated in a pulsed beam with spill duration longer than the usual few milliseconds: photographs will only be taken when an event occurs in the pressure minimum of the chamber's expansion, resulting in tracks with desired bubble density;

- to suppress picture taking when more than one event occurred during the sensitive time of the chamber, or

- to mark several events in one expansion cycle, to record their appearance on tape, which permits to distinguish later between neutrino interactions and non-related, out-of-time neutron stars;

- to produce a constant delay between the occurrence of the event and its photographic recording, in particular when short and precise delays for holography are envisaged;

- to operate a gate for the charge collection read-out, thus suppressing unwanted electronic noise, caused by mechanical vibrations, and signals from out-oftime particles;

- to mark events instantaneously with the help of laser-induced bubble strings: these bubbles are subject to almost identical growth and displacement as those on particle tracks, thus checking the chamber's sensitivity and turbulences at the occurrence of the event.

\section{Beam extraction and event rates}

Some scenarios for extraction from the Superconducting Super Collider (SSC) have been discussed [12] and will be reported briefly. The SSC may store up to $2 \times 10^{14}$ protons per ring. Since the collider will probably dump the old beam and refill every twelve hours or so, two extraction modes can be envisaged, which will have essentially no effect on the collider program:

(a) Twice per day $\sim 10^{14}$ protons can be dumped over some $100 \mathrm{~s}$ with slow parasitic extraction.

(b) Since a single turn abort system for $<2 \times 10^{14}$ protons has to be provided for safety reasons, it could serve at the same time as a source for beam dump neutrinos.

There are mainly two other extraction modes, which are not compatible with simultaneous collider operation: (c) A dedicated slow spill could eject as many as 2 spills $/ \mathrm{h}(600 \mathrm{~s}$ injection, $500 \mathrm{~s}$ ramp, $500 \mathrm{~s}$ ramp down) with $2 \times 10^{14}$ protons over $\sim 200 \mathrm{~s}$.

(d) A dedicated ping beam would distribute the proton intensity more evenly in time. One could have -100 pings $/ \mathrm{h}$ of $3 \mu$ s duration, with $2 \times 10^{11} \mathrm{p} /$ ping.

Alternative extraction modes, such as:

(e) secondaries from a high luminosity colliding beam region,

(f) extraction using a gas jet target [13],

(g) stochastic resonant extraction,

(h) synchrotron radiation extraction,

will not be considered in the present context, because they give either not enough intensity for bubble chamber experiments and/or are not yet sufficiently investigated.

Methods (a), (c), (d) and to a lesser extent (b), are all suitable for neutrino counter experiments, since the mass of the detector can be adapted to the beam intensity and its time structure (long flat top or rapid pings) or vice-versa. Bubble chambers, however, work best in a pulsed beam, and in general they can neither take more than one event per photograph, nor can they be built extremely long to compensate for a low neutrino flux. Therefore, a simultaneous operation of counter experiments and a bubble chamber in the same beam line at the SSC might be difficult and proton cost-inefficient.

A choice between the various extraction modes and beams is correlated with the size and performance of the bubble chamber. We have to fix tentatively dimensions and operation mode: both will be subject to modifications when the performance parameters of the accelerator are better known.

The chamber should be a cylinder (for physics and mechanical reasons), with its axis centred along the beam direction, $\sim 10 \mathrm{~m}$ long and $\sim 2 \mathrm{~m}$ in diameter. A schematic side view is given in fig. 1. Optics should be on one side and scintillation detectors on either side of the cylindrical vessel, allowing for stereo-reconstruction and timing of events. The expansion system is on its bottom. The first third of the volume is the vertex region, which means that only events having the origin in this part are considered for evaluation. The remainder of the downstream volume is needed for calorimetry measurements $(\sim 9$ nuclear collision lengths for full containment of the showers) as well as for muon identification in these events.

Neutrinos produced by multi-TeV protons are strongly collimated in a forward cone, so that $-90 \%$ interact in a detector with $0.5 \mathrm{~m}$ radius. Here we assumed that the detector, in the case of a bare target beam with $4 \mathrm{~km}$ decay length, is just behind the $2 \mathrm{~km}$ long shield, and in the case of the beam dump $1 \mathrm{~km}$ behind the dump area. With the above assumptions the useful interaction mass is $\leqslant 3 \mathrm{t}$ out of a total of $\sim 30 \mathrm{t}$ ). We do not aim for more than $\sim 0.3$ interactions/ 


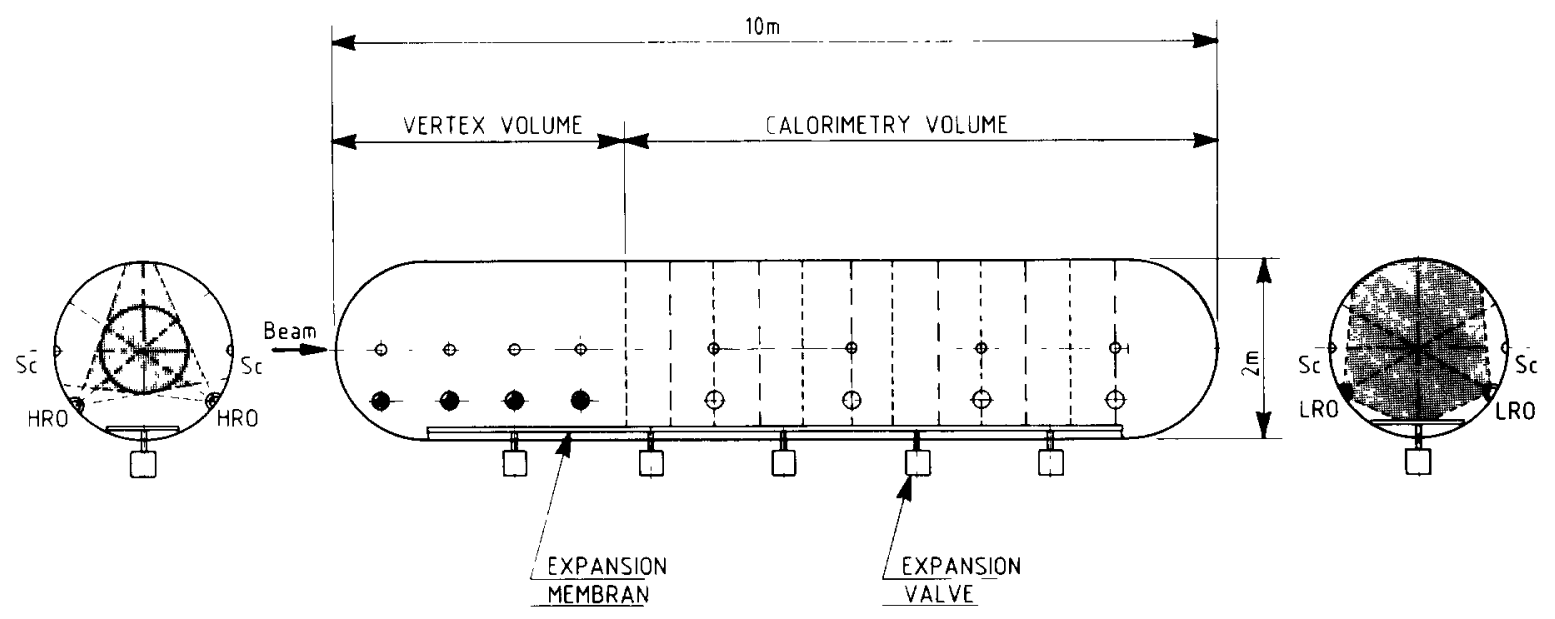

Fig. 1. Schematic layout of an argon bubble chamber/calorimeter. Longitudinal and transversal cross section: $\bullet$ high resolution optics (HRO, $\sim 60^{\circ}$ total acceptance) or holography; $O$ low resolution optics (LRO, $\sim 110^{\circ}$ total acceptance); shaded: overlapping visible region of two cameras at intersection of cones; - Scintillation detector, Sc; - - - Ionization collection electrodes; - - - Grounded grids.

expansion in the vertex volume. Using the calculations of Mori [14] (presently being updated by Morfin [15]), we can expect at $20 \mathrm{TeV}$ proton energy and $10^{14}$ incident protons in the case of a bare target beam $\sim 222 \nu_{\mu}$ interactions, and in the case of the dump $-11.4 \nu_{\mathrm{e}}$ and $\sim 0.36 \nu_{\tau}$ interactions.

Experience on repetition rate and sensitive time is limited to our small 31 argon bubble chamber. Any estimate of the dynamic heat load, which determines the above parameters for a much larger chamber, is difficult. It is dependent upon the potential sources of parasitic boiling, such as window flanges for optics and scintillation detection and the grids for charge collection. A high pressure drop during expansion is compulsary to obtain good track quality and sufficiently high bubble density. As a working hypothesis we take a repetition rate of -1 expansion in $3 \mathrm{~s}$, and hope to obtain with a servo-controlled hydraulic expansion system a pressure stabilized sensitive time of $\leqslant 30 \mathrm{~ms}$, i.e. a duty cycle which may approach an upper value of $\sim 1 \%$. Table 1 gives the event rates for these conditions, assuming for event rates/day an overall efficiency of $\frac{2}{3}$ for accelerator and detector.

The bubble chamber exposure by a dedicated ping beam (d) is almost ideal. The event rate is impressive, both for bare target beam and for a beam dump arrangement. However, the intensity per ping might be somewhat too low for counter experiments. A dedicated slow spill operation (c) is on the very limit of interest and the chamber would be run only if its operation costs are low. The single turn abort (b) would swamp the chamber completely with events, and poses for the dump itself tremendous technical problems. Slow para- sitic extraction (a) is useless for bubble chamber experiments.

Further studies of other extraction techniques are desirable. With the addition of some cryogenic and explosion proof equipment the chamber could also be operated with hydrogen, deuterium or nitrogen - all without the scintillation and charge collection features. Event rates can be roughly scaled from table 1 by the density ratios.

Table 1

Expected event rates in the fiducial volume of the proposed argon chamber for various extraction modes and beam layouts

\begin{tabular}{|c|c|c|c|}
\hline $\begin{array}{l}\text { Extraction } \\
\text { mode }\end{array}$ & Beam & $\begin{array}{l}\text { Events/ } \\
\text { expansion }\end{array}$ & $\begin{array}{l}\text { Events/ } \\
\text { day }\end{array}$ \\
\hline $\begin{array}{l}\text { (a) Slow parasitic } \\
\text { extraction } \\
2 \text { spills } / \mathrm{d} \\
-10^{14} \mathrm{p} / 100 \mathrm{~s}\end{array}$ & $\begin{array}{l}\text { bare target } \\
\text { dump }\end{array}$ & $\begin{array}{l}0.067 \nu_{\mu} \\
0.0035 \nu_{\mathrm{e}} \\
0.0001 \nu_{\tau}\end{array}$ & $\begin{array}{l}0.090 \nu_{\mu} \\
0.0046 \nu_{\mathrm{e}} \\
0.0002 \nu_{\tau}\end{array}$ \\
\hline $\begin{array}{l}\text { (b) Single turn abort } \\
2 \text { aborts } / \mathrm{d} \\
<2 \times 10^{14} \mathrm{p} / 3 \mu \mathrm{s}\end{array}$ & dump & $\begin{array}{l}22.8 \nu_{\mathrm{e}} \\
0.72 \nu_{\tau}\end{array}$ & $\begin{array}{l}30.4 \nu_{\mathrm{e}} \\
0.96 \nu_{\tau}\end{array}$ \\
\hline $\begin{array}{l}\text { (c) Dedicated slow } \\
\text { spill } \\
48 \text { spills } / \mathrm{d} \\
\leq 2 \times 10^{14} \mathrm{p} / 200 \mathrm{~s}\end{array}$ & $\begin{array}{l}\text { bare target } \\
\text { dump } \\
0.0001 \nu_{\tau}\end{array}$ & $\begin{array}{l}0.067 \nu_{\mu} \\
0.0035 \nu_{\mathrm{e}} \\
0.003 \nu_{\tau}\end{array}$ & $\begin{array}{l}2.15 \nu_{\mu} \\
0.11 \nu_{\mathrm{e}}\end{array}$ \\
\hline $\begin{array}{l}\text { (d) Dedicated ping } \\
1200 \text { pings } / \mathrm{h} \\
19200 \text { pings } / \mathrm{d} \\
\leq 1.6 \times 10^{11} \mathrm{p} / 3 \mu \mathrm{s}\end{array}$ & $\begin{array}{l}\text { bare target } \\
\text { dump }\end{array}$ & $\begin{array}{l}0.37 \nu_{\mu} \\
0.019 \nu_{\mathrm{e}} \\
0.0006 \nu_{\tau}\end{array}$ & $\begin{array}{l}4740 \nu_{\mu} \\
243 \nu_{\mathrm{e}} \\
7.67 \nu_{\tau}\end{array}$ \\
\hline
\end{tabular}




\section{Bubble chamber design}

The basic principle of an argon bubble chamber/ calorimeter/scintillation detector has been tested successfully on a small scale. The design of a multi-ton argon bubble chamber should be preceded by detailed Monte-Carlo calculations on particle multiplicities and of lateral and transversal shower development in the presence of a magnetic field. They will determine the aspect ratio of the chamber. The required optical resolution in the vertex region should be estimated, followed by further tests of holography [16]. Maximum obtainable drift distances and structure of the charge collection electrodes have to be investigated. Scintillation detectors have to be electronically interconnected such that the signal height is proportional to the number of tracks in each predetermined segment of the chamber. An expansion system should be able to stabilize the expanded pressure over several tens of milliseconds. A large chamber of this type can be built, but requires important engineering efforts.

\section{References}

[1] G. Harigel, H. Kautzky, P. McIntyre and A. van Ginneken, ARGONAUT, A Novel Detector for Very High Energy Neutrino Interactions, Fermilab Proposal 601 (1978).

[2] G. Harigel, G. Linser and F. Schenk, Nucl. Instr. and Meth. 187 (1981) 363 .
[3] G. Hangel, H.J. Hilke, G. Linser and F. Schenk, Nucl. Instr. and Meth. 188 (1981) 517.

[4] J. C. Berset, M. Burns, G. Harigel, J. Lindsay, G. Linser and F. Schenk, Nucl. Instr. and Meth. 203 (1982) 141.

[5] J.C Berset, M. Burns, K. Geissler, G. Harigel, J. Lindsay, G. Linser and F. Schenk, Nucl. Instr. and Meth. 203 (1982) 133.

[6] G. Harigel, Proc. Int. Europhysics Conf. on High Energy Physics, Brighton (1983) p. 421.

[7] G. Harigel, Nucl. Instr. and Meth. 225 (1984) 637

[8] G. Harigel, in Proc. SSC Fixed Target Workshop, Woodlands, Texas (1984) p. 48.

[9] G. Harigel, A. Hervé and K. Winter, Nucl. Instr. and Meth. 216 (1984) 355.

[10] P. Musset, Second ICFA Workshop on Possibilities and Limitations of Accelerators and Detectors, Les Diablerets, Switzerland (October 1979) p. 342.

[11] A. deRujula, to be published in Nucl. Instr. and Meth.

[12] A. Bodek, D. Crossairt, R. Huson, E. Keil, W. Wenzel and M. Zisman, in Proc. SSC Fixed-Target Workshop, Woodlands, Texas (1984) p. 14.

[13] W.A. Wenzel, in Proc. SSC Fixed-Target Workshop, Woodlands, Texas (1984) p. 15.

[14] S. Mor1, Proc. Second ICFA Workshop on Possibilities and Limitations of Accelerators and Detectors, Les Diablerets, Switzerland (October 1979) p. 333.

[15] J.G. Morfin, in Proc. SSC Fixed-Target Workshop, Woodlands, Texas (1984) p. 24.

[16] H. Bjelkhagen, G. Harigel, F. Pouyat, W. Seidl, C. Baltay, M. Bregman, F. Eisler, M. Hibbs, A. Schaffer, B. Cence, B. Brucker and T. Hart, Nucl. Instr. and Meth. 227 (1984) 437. 